\title{
ML277 specifically enhances pore opening of KCNQ1 with VSD at the activated state by modulating VSD-pore coupling
}

\section{Short title: ML277 enhances the VSD-pore coupling of KCNQ1}

\author{
Panpan Hou ${ }^{1}$, Jingyi Shi ${ }^{1}$, Kelli McFarland White ${ }^{1}$, Yuan Gao ${ }^{2}$, Jianmin Cui ${ }^{1, *}$
}

${ }^{1}$ Department of Biomedical Engineering, Center for the Investigation of Membrane Excitability Disorders, and Cardiac Bioelectricity, and Arrhythmia Center, Washington University, St. Louis, MO 63130, USA

${ }^{2}$ Tencent Al Lab, Shenzhen 518052, China.

${ }^{*}$ Correspondence: jcui@wustl.edu (J.C.) 


\section{Abstract}

In response to membrane depolarization, the KCNQ1 potassium channel opens at the intermediate $(\mathrm{IO})$ and activated $(\mathrm{AO})$ states that correspond to the stepwise activation of the voltage sensing domain (VSD) to the intermediate (I) and activated (A) states. In the heart, KCNQ1 associates with the auxiliary subunit KCNE1 to form the $I_{\mathrm{Ks}}$ channel that regulates heart rhythm. More than 300 of loss-of-function KCNQ1 mutations cause long QT syndrome (LQTS). KCNE1 suppresses the IO state so that the $I_{\mathrm{Ks}}$ channel opens only to the $A O$ state. Thus, enhancing $A O$ state presents a potential therapy for antiLQTS. Here, we systematically tested modulations of KCNQ1 channels by a KCNQ1 activator, ML277. It enhances the current amplitude, slows down activation, deactivation and inactivation kinetics, shifts the voltage dependence of activation to more positive voltages, decreases the $\mathrm{Rb}^{+} / \mathrm{K}^{+}$permeability ratio, and selectively increases currents of mutant KCNQ1 channels that open only to the AO state. All these observations are consistent with the mechanism that ML277 specifically potentiates the AO state. On the other hand, ML277 does not affect the VSD activation, suggesting that it potentiates the AO state by enhancing the electromechanical (E-M) coupling when the VSD moves to the activated state. Our results suggest that ML277 provides a unique tool to investigate the gating mechanism of KCNQ1 and I $\mathrm{Ks}_{\mathrm{s}}$ channels. The specificity of ML277 to increase the $A O$ state of native $I_{\mathrm{Ks}}$ currents also suggests a new strategy for anti-LQTS therapy. 


\section{Introduction}

KCNQ1, also known as $K_{V} 7.1$ or $K_{V} L Q T 1$, belongs to a subfamily of voltage-activated potassium channels (Barhanin et al., 1996; Sanguinetti et al., 1996; Wang et al., 1996). It adopts the canonical structural organization of the homo-tetrameric $K_{v}$ superfamily, in which each subunit contains six transmembrane segments (S1-S6), with the first four (S1-S4) forming the voltage sensing domain (VSD) and the latter two (S5-S6) folding to the pore (Sun and MacKinnon, 2017). In response to membrane depolarization, the S4 segment in the VSD undergoes outward movement (Glauner et al., 1999; Larsson et al., 1996), and triggers pore opening via electromechanical (E-M) coupling interactions between the VSD and the pore. Similar to other $K_{V}$ members such as the Shaker channel, the VSD of KCNQ1 channels shows two steps activation: it activates sequentially from the resting state to an experimentally resolvable intermediate state before finally arriving at the fully activated state (Baker et al., 1998; Barro-Soria et al., 2014; Bezanilla et al., 1994; Carvalho-de-Souza and Bezanilla, 2018; Delemotte et al., 2011; Hou et al., 2017; Jensen et al., 2012; Lacroix et al., 2012; Ledwell and Aldrich, 1999; Osteen et al., 2012; Sigg et al., 1994; Sigworth, 1994; Silva et al., 2009; Silverman et al., 2003; Wu et al., 2010a; Wu et al., 2010b; Zagotta et al., 1994; Zaydman et al., 2014). However, unlike the Shaker channel which opens only when the VSD moves to the fully activated state, the KCNQ1 channel opens when its VSD occupies both the intermediate and fully activated conformations, resulting in intermediate-open (IO) and activated-open (AO) states (Hou et al., 2017; Zaydman et al., 2014).

The two open states, 10 and $A O$, are distinctive from each other. Structurally, they associate with different VSD and pore conformations. During membrane depolarization, the S4 segment moves outward from its resting state to the intermediate state to trigger the 10 state opening. Subsequent movement of the S4 helix from the intermediate state to the activated state then triggers conformational change of the pore from the $1 \mathrm{O}$ state to the AO state. These VSD-pore conformations can be isolated by the mutation that arrests the VSD at the intermediate state, E160R/R231E (E1R/R2E), which results in the constitutive IO state, and the mutation that arrests the VSD at the activated state, 
E160R/R237E (E1R/R4E), which results in the constitutive AO state (Restier et al., 2008; Wu et al., 2010a; Wu et al., 2010b; Zaydman et al., 2014). Functionally, the two open states have different gating properties and are differentially modulated by the auxiliary subunit KCNE1, allowing us to experimentally distinguish them. These differences include (1) the AO state shows slower current kinetics and more positive voltage-dependent activation than that of the IO state, (2) KCNQ1 channels show lower open probability when the VSD moves from the intermediate state to the fully activated state, resulting in inactivation phenotypes under certain voltage stimulations, (3) the AO state shows lower $\mathrm{Rb}^{+} / \mathrm{K}^{+}$permeability ratio than the $\mathrm{IO}$ state, (4) different $\mathrm{E}-\mathrm{M}$ coupling mechanisms such that the two open states can be isolated by single mutations that ablate respective E-M couplings, and (5) KCNE1 suppresses the IO state but enhances the AO state (Hou et al., 2017; Zaydman et al., 2014).

In cardiomyocytes, KCNQ1 associates with KCNE1 to generate the slow delayed rectifier current $\left(\mathrm{I}_{\mathrm{Ks}}\right)$ important for terminating cardiac action potentials (Barhanin et al., 1996; Chiamvimonvat et al., 2017; Keating and Sanguinetti, 2001; Sanguinetti et al., 1996). Clinically, more than 300 loss-of-function mutations in KCNQ1 have been found to induce functional defects to the $I_{\mathrm{Ks}}$ current, causing long QT syndrome (LQTS) that predisposes patients to life-threatening cardiac arrhythmia (Schwartz et al., 2012; Wang et al., 1996). KCNE1 specifically suppresses the IO state but enhances the AO state, rendering the $\mathrm{I}_{\mathrm{Ks}}$ channel with only AO state openings (Zaydman et al., 2014). Therefore, the AO state is physiologically more important in the heart. Enhancing AO state currents to specifically rescue the functional defects of the mutant $I_{K s}$ currents presents a potential strategy for anti-LQTS therapy.

Different activators have been found to enhance KCNQ1 and $I_{K s}$ currents. Zinc pyrithione (ZnPy), L-364,373 (R-L3), 4,4'-diisothiocyanatostilbene-2,2'-disulfonic acid (DIDS), and mefenamic acid (MFA) activate either homomeric KCNQ1 or KCNQ1+KCNE1 complex (Abitbol et al., 1999; Busch et al., 1997; Gao et al., 2008; Salata et al., 1998; Seebohm et al., 2003a; Xiong et al., 2007), while phenylboronic acid (PBA), Hexachlorophene (HCP), mallotoxin (MTX), and 3-ethyl-2-hydroxy-2cyclopenten-1-one (CPT1) potentiate both KCNQ1 and KCNQ1+KCNE1 channels (De 
Silva et al., 2018; Mruk and Kobertz, 2009; Zheng et al., 2012). Although some of these activators are capable of shortening the action potential duration (APD) in cardiac myocytes (Salata et al., 1998; Zheng et al., 2012), none of them was found to only enhance the AO state of KCNQ1 or $I_{\mathrm{Ks}}$ channels. Besides KCNQ1 and $I_{\mathrm{Ks}}$ channels, all these activators also strongly modulate other ion channels that are key to cardiac electrophysiology, such as hERG, $\mathrm{Ca}_{\mathrm{v}} 1.2$, and $\mathrm{Na}_{\mathrm{v}} 1.5$ in the heart and neuronal KCNQ channels (KCNQ2-KCNQ5) (Cruickshank et al., 2003; De Silva et al., 2018; Gao et al., 2017; Hill and Sitsapesan, 2002; Mruk and Kobertz, 2009; Salata et al., 1998; Zheng et al., 2012). The low specificity may cause severe side effects to LQTS patients, limiting the potential for further development into anti-LQTS drugs.

On the other hand, a recently identified KCNQ1 activator ML277 shows potent effect to KCNQ1 channel, while demonstrating little effects on other important cardiac ion channels including hERG, Nav1.5, and Cav1.2 channels, and other KCNQ isoforms ( $\mathrm{Yu}$ et al., 2013; Yu et al., 2010). Moreover, ML277 shortens the APD of human induced pluripotent stem cell (iPSC) derived cardiomyocytes and guinea pig cardiomyocytes, via activating the $\mathrm{I}_{\mathrm{Ks}}$ currents (Xu et al., 2015; Yu et al., 2013). The high specificity and potent potentiation in native $\mathrm{I}_{\mathrm{Ks}}$ currents make ML277 a promising anti-LQTS drug candidate. Although computational and experimental studies have been performed to probe the binding and potentiation of ML277 on KCNQ1 and $I_{\mathrm{Ks}}$ channels ( $\mathrm{Xu}$ et al., 2015; Yu et al., 2013), the mechanism underlying this potentiation on the two open states of KCNQ1 channels remains largely unclear.

In this study, we find that ML277 changes KCNQ1 channel current kinetics, alters voltage dependence of activation, inactivation property, and ion permeation. All these modifications are consistent with the mechanism that ML277 specifically enhances the current of the AO state. Supporting this mechanism, ML277 selectively enhances the currents of mutant KCNQ1 channels that open only at the AO state but not the mutant channels that open only at the IO state. These results and recordings of VSD activation using voltage clamp fluorometry (VCF) suggest that ML277 specifically promotes the E$M$ coupling when the VSD is at the activated state to increase the AO state current. KCNQ1 is the first ion channel that shows well resolved two open states when the VSD is at different activation states. This study sets the first example that a small molecule 
compound can specifically modulate only one of the two open states via enhancing the specific E-M coupling. Our study not only proposes an effective tool to investigate the gating mechanism of KCNQ1 channel with two open states, but also paves the way to new strategies, enhancing the $\mathrm{AO}$ state of native $\mathrm{I}_{\mathrm{Ks}}$ currents, for treating LQTS.

\section{Results}

\section{ML277 specifically enhances currents of the AO state}

Previous studies have shown that, in Chinese hamster ovary (CHO) cells, ML277 potentiates both KCNQ1 channels and KCNQ1+KCNE1 channels with unsaturated KCNE1 association. Progressive increase of KCNE1 expression reduces efficacy of ML277 and eventually abolishes its effect on KCNQ1+KCNE1 channels with saturated KCNE1 binding (Yu et al., 2013). We tested the ML277 effects on KCNQ1 and $\mathrm{I}_{\mathrm{Ks}}$ channels expressed in Xenopus oocytes, and found that $1 \mu \mathrm{M}$ ML277 increased the KCNQ1 current amplitudes, such that the same depolarizing voltage elicited larger currents (Figure 1A), while $1 \mu \mathrm{M}$ ML277 showed less current increase on KCNQ1+KCNE1 channels with injected mRNA weight ratio of KCNQ1:KCNE1=300:1, which may induce unsaturated KCNE1 binding, and showed no effect on KCNQ1+KCNE1 channels with injected mRNA weight ratio of $4: 1$, which may saturate the KCNE1 binding (Figure 1B) (Nakajo et al., 2010). These results recorded from Xenopus oocytes are consistent with previous findings from $\mathrm{CHO}$ cells (Yu et al., 2013). The loss of ML277 effects on KCNQ1+KCNE1 channels with saturating KCNE1 association was suggested to result from a competition between ML277 and KCNE1 in binding to KCNQ1 (Yu et al., 2013).

The KCNQ1 currents can be fitted with two distinct exponential components in response to depolarization pulses (Figure 1A) (Hou et al., 2017). The fast time course approximates the channels opening to the 10 state, while the slow component is mainly determined by the channels opening to the AO state (Hou et al., 2017). ML277 increased only the slow current component, while leaving the fast current component unaltered, which is clearly shown by merging the currents before and after ML277 application (Figure 1A,C). We compared the time constants of the fast and slow components before and after ML277 application. For example, at $+40 \mathrm{mV}$ depolarization, 
the time constant of the slow component $\left(\tau_{\mathrm{s}}\right)$ was changed from $0.57 \mathrm{~s}$ to $0.91 \mathrm{~s}$, while the time constant of the fast component $\left(\tau_{f}\right)$ changed little (from 45 ms to $47 \mathrm{~ms}$, Figure 1C). This selective change on the $\tau_{\mathrm{s}}$ was observed in different voltages (Figure 1D). A change only in $\tau_{\mathrm{s}}$ but not $\tau_{\mathrm{f}}$ is quantitatively consistent with the mechanism that ML277 selectively alters the $\mathrm{AO}$ state without affecting the $\mathrm{IO}$ state.

Our previous studies showed that the $\mathrm{IO}$ and $\mathrm{AO}$ states have different time- and voltage-dependence: the AO state appears only when the KCNQ1 channel is activated by voltages above $-40 \mathrm{mV}$ and for a sufficient time duration ( $\geq 100 \mathrm{~ms}$ at $+40 \mathrm{mV}$ ) (Hou et al., 2017). To verify that ML277 selectively increases the currents of the AO state, we tested the effect of $1 \mu \mathrm{M}$ ML277 on KCNQ1 current when we activated KCNQ1 channels either at low voltage $(-50 \mathrm{mV})$ or at high voltage with a short time duration (+40 mV for only $100 \mathrm{~ms}$ ). These voltage protocol effectively restricts KCNQ1 channel activation to the 10 state. In both cases, ML277 did not increase current amplitudes or alter current kinetics (Figure 1E,F). These data support that ML277 specifically modifies the current of the AO state.

\section{ML277 right shifts the G-V relationship of KCNQ1 channels}

We analyzed ML277 induced changes in the voltage-dependent activation of KCNQ1 channels. ML277 activated the channel and nearly doubled the current size and the total conductance (Figures $1 \mathrm{~A}$ and $2 \mathrm{~A}$ ). Interestingly, unlike other activators that potentiate ion channel currents by left-shifting the $\mathrm{G}-\mathrm{V}$ relationship (De Silva et al., 2018; Gao et al., 2017; Guo et al., 2017; Liu et al., 2013; Seebohm et al., 2003a; Zheng et al., 2012), ML277 slightly shifted the G-V relationship of KCNQ1 channels to more positive voltages $(\Delta \mathrm{V} \sim 6 \mathrm{mV}$, Figure $2 \mathrm{~B}, \mathrm{C})$. This curious result can be explained by properties of KCNQ1 voltage-dependent activation process. The KCNQ1 currents are composed of currents of both the $\mathrm{IO}$ and $\mathrm{AO}$ states; therefore, the $\mathrm{G}-\mathrm{V}$ relation of KCNQ1 channels is the sum of the $\mathrm{G}-\mathrm{V}$ relations of both the $\mathrm{IO}$ and $\mathrm{AO}$ states. The $\mathrm{G}-\mathrm{V}$ relationship of the $\mathrm{AO}$ state is at more positive voltages compared to the $1 \mathrm{O}$ state, reflecting the fact that the activated VSD state requires more positive voltages than the intermediate VSD state (Hou et al., 2017; Zaydman et al., 2014). Our previous studies demonstrated that KCNQ1 conducts current predominately through the IO state (Hou et 
al., 2017; Zaydman et al., 2014). This property is also manifested in the kinetics of current activation, in which the slow component (primarily through the $\mathrm{AO}$ state) accounts for only a small portion of the total current as compared to the fast component (primarily through the $\mathrm{IO}$ state) (Figure $1 \mathrm{~A}$ ). The $\mathrm{G}-\mathrm{V}$ relation of KCNQ1 channels thus tracks closely to the $\mathrm{G}-\mathrm{V}$ relation of the $\mathrm{IO}$ state, and contains a small portion of the $\mathrm{G}-$ $\mathrm{V}$ relation of the $\mathrm{AO}$ state. By contrast, the $\mathrm{I}_{\mathrm{Ks}}(\mathrm{KCNQ1+KCNE1)}$ channel conducts predominantly with AO-state current, because KCNE1 suppresses pore conductance of the IO state (Zaydman et al., 2014). The G-V curve of the $I_{K s}$ channel is thus dominated by the $\mathrm{G}-\mathrm{V}$ relationship of the $\mathrm{AO}$ state, and is significantly right-shifted compared to the KCNQ1 G-V relationship (Figure 2C,D). The contrasting G-V relations of the KCNQ1 (IO-dominant) and $\mathrm{I}_{\mathrm{Ks}}$ (AO-dominant) are illustrated in Figure $2 \mathrm{C}$ and $\mathrm{D}$. In this study, we utilized a single Boltzmann function, which assumes one closed state and one open state, to approximate the complex KCNQ1 G-V relation (Figure 2A,B). Accordingly, if ML277 increases the contribution of the AO state to the overall current, then a right-shift in the G-V relationship is expected, as the $\mathrm{AO}$ state is right-shifted compared to the 10 state. We indeed observed a small right-shift in the KCNQ1 G-V relationship upon ML277 application (Figure 2B,C). The right shift of the G-V relation by ML277 is consistent with the mechanism that ML277 selectively increases the AO state currents and thereby increases contribution of the $A O$ state $\mathrm{G}-\mathrm{V}$ relation to the overall $\mathrm{G}-\mathrm{V}$ curve.

\section{ML277 changes the inactivation of KCNQ1 channels}

KCNQ1 channels show an incomplete inactivation that can be observed by a hook in tail currents at hyperpolarized voltages after a pre-pulse that activates the channel (Abitbol et al., 1999; Gibor et al., 2007; Larsen et al., 2011; Tristani-Firouzi and Sanguinetti, 1998). The hook is due to an initial increase of the inward (downward) tail current followed by continuous decay of the current (Figure 3A), consistent with the idea that channels recover from inactivation upon hyperpolarization via an open state before closing due to deactivation (Gibor et al., 2007; Larsen et al., 2011; Pusch et al., 1998; Seebohm et al., 2003b). Recently we found that the so-called inactivation state in KCNQ1 channels is the AO state, and the hook current reflects channel deactivation from the $A O$ state to the $1 O$ state because the channels have a higher open probability 
when the VSD is at the intermediate state than at the activated state (Hou et al., 2017). If ML277 selectively enhances the currents through the AO state, we expect to observe a change in KCNQ1 hook currents.

We recorded tail currents during hyperpolarization to $-120 \mathrm{mV}$, with varying time durations of pre-pulses at $+40 \mathrm{mV}$ that activated KCNQ1 channels. The hook current developed with pre-pulse time durations, and saturated at the $2.9 \mathrm{~s}$ pre-pulse (red, Figure $3 \mathrm{~A}$ ). To quantify the inactivation, we fitted the tail currents with a double exponential function $F(t)=A_{1} \times \exp \left(-t / \tau_{1}\right)+A_{2} \times \exp \left(-t / \tau_{2}\right)+C$ (Figure 3B), where $A_{1}$ and $A_{2}$ are amplitudes, $\tau_{1}$ and $\tau_{2}$ are time constants, and $C$ is the offset due to leak currents. The hook current, recorded after a pre-pulse of $2.913 \mathrm{~s}$ duration at $+40 \mathrm{mV}$ (red, Figure $3 A, B$ ), was well fitted by the double exponential function (green dots, Figure 3B). $A_{1} \times \exp \left(-t / \tau_{1}\right)+C$ represents the deactivation process (green line, Figure $3 \mathrm{~B}$ ), and $A_{2} \times \exp \left(-t / \tau_{2}\right)$ represents the hook current (green line, Figure 3B). $A_{2}$ is the amplitude of the hook current, which is used to quantify the KCNQ1 inactivation. The normalized $A_{2}$ before (black) and after (blue) adding ML277 at varying pre-pulse time durations is shown in Figure 3D. It shows that ML277 slows down the development of $A_{2}$ (from 0.56 $\mathrm{s}$ to $1.02 \mathrm{~s}$, Figure 3D), consistent with the effect that ML277 prolongs the development of the $\mathrm{AO}$ state (from $0.57 \mathrm{~s}$ to $0.91 \mathrm{~s}$, Figure 1B). These results reveal that ML277 modulates the $\mathrm{AO}$ state and thus the inactivation property.

\section{ML277 changes ion permeation of KCNQ1 channels}

Previous studies found that $K C N Q 1$ and $I_{K s}$ channels have different ion permeation. The inward tail currents of KCNQ1 channels at hyperpolarized voltages are increased about threefold upon substitution of $100 \mathrm{mM}$ potassium with $100 \mathrm{mM}$ rubidium (Pusch et al., 2000), however, KCNE1 association reverses this change and makes the $I_{K s}$ channel conducts less tail current in $100 \mathrm{mM}$ rubidium than in $100 \mathrm{mM}$ potassium (Pusch et al., 2000; Seebohm et al., 2003b). We recently found that these results could be explained by the mechanism that the $\mathrm{IO}$ and $\mathrm{AO}$ states have different $\mathrm{Rb}^{+} / \mathrm{K}^{+}$permeability ratio (Zaydman et al., 2014). Using E1R/R2E and E1R/R4E mutations that open in the IO and $A O$ state, respectively, we found that $E 1 R / R 4 E$ had a significantly lower $\mathrm{Rb}^{+} / \mathrm{K}^{+}$permeability ratio compared to $\mathrm{E} 1 \mathrm{R} / \mathrm{R} 2 \mathrm{E}$ channels, indicating that the $\mathrm{AO}$ state 
has a lower $\mathrm{Rb}^{+} / \mathrm{K}^{+}$permeability ratio than the $\mathrm{IO}$ state (Zaydman et al., 2014). Since KCNQ1 channels open predominately to the $I O$ state, they show a high $\mathrm{Rb}^{+} / \mathrm{K}^{+}$permeability ratio, similar to that of the $\mathrm{IO}$ state. On the other hand, since KCNE1 suppresses the $1 O$ state but enhances the $A O$ state, the $I_{K s}$ channels show a lower $\mathrm{Rb}^{+} / \mathrm{K}^{+}$permeability ratio (Zaydman et al., 2014).

To test ML277 induced changes in ion permeation of KCNQ1 channels, we measured tail current amplitudes of the channels before and after adding $1 \mu \mathrm{M} \mathrm{ML277}$ at $-60 \mathrm{mV}$ after a $+60 \mathrm{mV}$ pre-pulse for $5 \mathrm{~s}$ in $100 \mathrm{mM} \mathrm{Rb}^{+}$and $100 \mathrm{mM} \mathrm{K}^{+}$solutions, respectively. ML277 significantly decreased the $\mathrm{Rb}^{+} / \mathrm{K}^{+}$permeability ratio of KCNQ1 channels (from 3.1 to 1.1 , Figure 4). The $\mathrm{Rb}^{+} / \mathrm{K}^{+}$permeability ratio of $\mathrm{KCNQ1}$ channels after treating ML277 is closer to that of the $I_{\mathrm{Ks}}$ channels (Figure 4B), which is consistent with previous finding (Xu et al., 2015). These results are consistent with the mechanism that ML277 selectively enhances currents of the $A O$ state to change the $1 O / A O$ composition in KCNQ1 channels, and the increased fraction of $\mathrm{AO}$ currents changes the $\mathrm{Rb}^{+} / \mathrm{K}^{+}$ permeability ratio towards that of the $\mathrm{AO}$ state.

\section{ML277 specifically increases the currents of mutant KCNQ1 channels that open only to the AO state}

In previous studies we have demonstrated that the $\mathrm{IO}$ and $\mathrm{AO}$ states can be isolated in mutant KCNQ1 channels. Two pairs of mutations make the channels open exclusively to the $\mathrm{IO}$ and $\mathrm{AO}$ states, respectively with different mechanisms. The first pair, E1R/R2E and $\mathrm{E} 1 \mathrm{R} / \mathrm{R} 4 \mathrm{E}$ arrest the VSDs at the intermediate and activated states, respectively, make the channels constitutively open at the $1 \mathrm{O}$ and $\mathrm{AO}$ states, respectively (Restier et al., 2008; Wu et al., 2010a; Wu et al., 2010b; Zaydman et al., 2014). The second pair, S338F and F351A disrupt the VSD-pore couplings when the VSDs are at the activated and intermediate states, respectively, make the channels open only to the $\mathrm{IO}$ and $\mathrm{AO}$ states, respectively (Boulet et al., 2007; Hoosien et al., 2013; Hou et al., 2017; Zaydman et al., 2014).

To confirm that ML277 specifically enhances the AO state current, we measured its effects on these mutant channels. ML277 increased currents of the mutant channels $\mathrm{E} 1 \mathrm{R} / \mathrm{R} 4 \mathrm{E}$ and F351A that open only in the AO state, but barely enhanced the mutant 
channels E1R/R2E or S338F that open only to the IO state (Figure 5). These results support the mechanism that ML277 selectively enhances the current of the AO state.

\section{ML277 enhances the E-M coupling specifically when the VSD is at the activated state}

The above results all support the mechanism that ML277 selectively enhances the currents of the AO state in KCNQ1 channels. Since ML277 slows down the time course of both activation and deactivation (Figure 1C, 3A,C), it is likely to modulate the gating mechanism of KCNQ1 that specifically controls the AO state. The voltage-dependent activation of a $\mathrm{K}_{\mathrm{V}}$ channel involves three molecular events: VSD activation upon transmembrane voltage depolarization, rearrangement in interactions between the VSD and the pore (E-M coupling), and the pore opening to conduct ionic current (Cui, 2016). Interestingly, ML277 enhances the constitutive current of E1R/R4E mutant channels (Figure 5A,C), in which the VSD is arrested at the activated state by the double charge reversal mutations (Zaydman et al., 2014). This result indicates that ML277 may enhance the coupling between the VSD and pore when the VSD is at the activated state to increase open probability.

To further study this mechanism, we carried out VCF experiments, a technique that VSD movements are monitored with a fluorophore attached to the S3-S4 linker in pseudo-WT KCNQ1 (KCNQ1-C214A/G219C/C331A) channels during activation while channel opening is monitored by current recordings (Barro-Soria et al., 2015; BarroSoria et al., 2014; Hou et al., 2017; Osteen et al., 2012; Osteen et al., 2010; Zaydman et al., 2014; Zaydman et al., 2013). The KCNQ1 fluorescence-voltage (F-V) relationship can be well-fitted by a double Boltzmann function, revealing the stepwise VSD activation processes (Figure 6A,B) (Hou et al., 2017; Zaydman et al., 2014). The timeand voltage-dependence of the VSD activation in the absence and presence of $1 \mu \mathrm{M}$ ML277 showed little change, and the fluorescence traces could be superimposed with each other at different voltages (Figure 6A,B). Similar to the WT KCNQ1 channels (Figure 2B), ML277 induced a right-shifted $\mathrm{G}-\mathrm{V}$ relationship in pseudo-WT KCNQ1 $(\Delta \mathrm{V} \sim 5 \mathrm{mV}$, Figure 6B). The VCF results indicate that ML277 does not affect the twostep VSD activation although it doubles the current size (Figure 6A). The unchanged 
VSD activation with doubled conductance in the AO state strongly suggests that ML277 enhances the E-M coupling to increase the $A O$ current. We have previously shown that the KCNQ1 channel may have distinct E-M coupling mechanisms to open the pore when the VSD is at the intermediate and activated state (Hou et al., 2017; Zaydman et al., 2014), thus small molecule compounds which can specifically target distinct E-M coupling pathways in KCNQ1 is not unexpected. Our results are consistent with the mechanism that ML277 may interact with a site in the KCNQ1 channel that controls E-M coupling specifically when the VSD is at the activated state.

The two open states gating processes of KCNQ1 channels can be illustrated in a scheme (Figure 6C) based on a simplified kinetic model without considering that the KCNQ1 channel is formed by four subunits with four identical VSDs (Hou et al., 2017; Zaydman et al., 2014). In this model, the resting closed (RC), intermediate closed (IC), and activated closed (AC) are VSD states at the resting, intermediate, and activated states when the pore is in the closed state, and $I O$ and $A O$ are the two open states. ML277 may specifically affect the E-M coupling of the AO state by stabilizing the conformational energy of the $\mathrm{AO}$ state (Figure 6C).

We parameterized this simple kinetic model and simulated the ML277 effects (Figure 7).

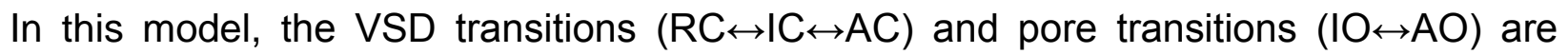
voltage dependent, and the VSD-pore coupling transitions (IC $\leftrightarrow I O$ and $A C \leftrightarrow A O)$ are voltage independent (see Methods). This model is capable of emulating the properties of the two open states in KCNQ1 channels, including the well resolved IO and AO states and the hooked tail currents at $-40 \mathrm{mV}$ repolarization voltage indicating the inactivation (black, Figure 7A) (Hou et al., 2017). In this model, $k_{2}$ and $\beta_{3}$ are the transition rates for $A O \rightarrow A C$ and $A O \rightarrow I O$ respectively. To model ML277's mechanism of enhancing $\mathrm{E}-\mathrm{M}$ coupling of the $\mathrm{AO}$ states, we reduced the rates of exiting from the $\mathrm{AO}$ state $\left(k_{2}\right.$ and $\left.\beta_{3}\right)$ by half, which corresponds to ML277 stabilizing the conformational energy of the AO state (Figure 7B). Strikingly, reducing these rates can recapitulate the ML277 effects on KCNQ1 channel, including the enhanced AO currents and the rightshifted G-V relationship (Figure 7A-D, see Methods). 
Taken together, our experimental and simulation results reveal that ML277 increases KCNQ1 current by promoting the AO state E-M coupling. This is a novel gating modification mechanism that may help understand the fundamental gating processes of KCNQ1 and $\mathrm{I}_{\mathrm{Ks}}$ channels, and develop new strategies for treating LQTS.

\section{Discussion}

In this work, we systematically studied the functional properties of KCNQ1 channels that are modulated by ML277, including current amplitudes and kinetics (Figure 1), voltage dependence of activation (Figure 2), inactivation (Figure 3), and ion permeation (Figure 4). All these modulations are consistent with the mechanism that ML277 specifically enhances the $A O$ state $E-M$ coupling to increase the $A O$ state current. Different compounds and toxins have been found as state-dependent modulators for ion channels. For instance, mexiletine preferentially blocks the Nav1.5 sodium channel when the DIII-VSD is activated, and protoxin-II shows higher affinity to resting VSD states of Nav1.7 sodium channel (Nakagawa et al., 2019; Xu et al., 2019; Zhu et al., 2019), while roscovitine facilitates the inactivation and shows less effect on activation of Cav1.2 calcium channel (Yarotskyy et al., 2010; Yazawa et al., 2011). We previously found that the 10 state is more sensitive to the blocker XE991 than the AO state (Zaydman et al., 2014). These modulators preferentially modulate one of the states of certain ion channels but still show considerable modulations at other states. In this study, we show a clean example of state-dependent modulation in which only the AO state of KCNQ1 channel is exclusively enhanced by ML277, leaving the IO state unaltered. This unique mechanism of gating modification reveals an interesting mechanistic feature of the KCNQ1 channel. Since only the AO state is exclusively modulated, this strongly suggests that there are structural and functional modules in KCNQ1 channels that engage in interactions exclusive for the AO state E-M coupling. Moreover, these interactions can be specifically altered, when the VSD is at the activated state, to yield higher open probability. This is consistent with our previous finding that single mutations can selectively disrupt the E-M couplings for the $\mathrm{IO}$ and $\mathrm{AO}$ states, respectively (Hou et al., 2017; Zaydman et al., 2014). In a previous study, Xu and colleagues proposed that ML277 intracellularly binds at the inter-subunit interface 
between intracellular loops (S2-S3 loop and S4-S5 linker) and the pore (Xu et al., 2015). It will be interesting to further study if this putative ML277 binding site is important in the E-M coupling for the $\mathrm{AO}$ state.

We studied 1 $\mu \mathrm{M}$ ML277 induced functional changes to KCNQ1 channels expressed in Xenopus oocytes. Some previous publications reported different observations under other experimental conditions. For example, Yu et al. found that in $\mathrm{CHO}$ cells, $0.3 \mu \mathrm{M}$ ML277 strongly increases KCNQ1 currents, left-shifts the G-V relationship, and abolishes inactivation (Yu et al., 2013). However, Xu et al. reported a much less effect of ML277 on KCNQ1 activation expressed in Xenopus oocytes than in CHO cells (Xu et al., 2015). Xu et al. also found that in Xenopus oocytes, 25 MM ML277 strongly decreases the $\mathrm{Rb}^{+} / \mathrm{K}^{+}$permeability ratio to the level even lower than $I_{\mathrm{Ks}}$ channels (Xu et al., 2015). The high concentrations of ML277 used by Xu and colleagues may enhance the $\mathrm{AO}$ state more than KCNE1 does to the channel, or the specificity of ML277 at this high concentration might have changed.

Activators of KCNQ1 and $\mathrm{I}_{\mathrm{Ks}}$ channels may be effective for treating LQTS. Although several KCNQ1 activators have been identified, their low specificity may limit further drug development (Cruickshank et al., 2003; De Silva et al., 2018; Gao et al., 2017; Hill and Sitsapesan, 2002; Mruk and Kobertz, 2009; Salata et al., 1998; Zheng et al., 2012). Several features of ML277 makes it an interesting KCNQ1 modulator. First, ML277 specifically activates KCNQ1 channel with little activity on other ion channels that are key to cardiac electrophysiology or neuronal KCNQ channels (Mattmann et al., 2012; Yu et al., 2013). Second, ML277 activates KCNQ1 channels expressed in mammalian cells with an $\mathrm{EC}_{50}$ of $260 \mathrm{nM}$ (Mattmann et al., 2012; Yu et al., 2013), lower than any other known KCNQ1// Ks activators. Finally, studies have confirmed that ML277 effectively shortens the APD in human iPSC derived cardiomyocytes (including LQTS conditions) and guinea pig cardiomyocytes (Ma et al., 2015; Xu et al., 2015; Yu et al., 2013). Interestingly, ML277 was found to increase only the current of KCNQ1 channels but not the $\mathrm{I}_{\mathrm{Ks}}$ channels with saturated KCNE1 association in exogenous expression (Figure 1A,B) (Yu et al., 2013). The sensitivity of $\mathrm{I}_{\mathrm{Ks}}$ currents to ML277 in cardiomyocytes suggests that native $\mathrm{I}_{\mathrm{Ks}}$ channels are not always saturated with KCNE1 
bioRxiv preprint doi: https://doi.org/10.1101/624478; this version posted May 2, 2019. The copyright holder for this preprint (which was not certified by peer review) is the author/funder. All rights reserved. No reuse allowed without permission.

association, and these native $\mathrm{I}_{\mathrm{Ks}}$ channels may be a novel target for compounds such as ML277 to treat LQTS. 


\section{Methods}

Constructs and mutagenesis

Overlap extension and high-fidelity PCR were used for making KCNQ1 channel point mutations. Each KCNQ1 mutation was verified by DNA sequencing. Then cRNA of WT KCNQ1 and mutants were synthesized using the mMessage T7 polymerase kit (Applied Biosystems-Thermo Fisher Scientific) for oocyte injections.

\section{Oocyte expression}

Oocytes (at stage $\mathrm{V}$ or $\mathrm{VI}$ ) were obtained from Xenopus laevis by laparotomy surgery, following the protocol approved by the Washington University Animal Studies Committee (Protocol \#20160046). Oocytes were then digested by collagenase (0.5 $\mathrm{mg} / \mathrm{ml}$, Sigma Aldrich, St Louis, MO) and micro-injected with KCNQ1 cRNAs. WT or mutant KCNQ1 cRNAs (9.2 ng) with or without KCNE1 cRNA (0.031 ng for KCNQ1:KCNE1 weight ratio of 300:1 or $2.3 \mathrm{ng}$ for KCNQ1:KCNE1 weight ratio of 4:1) were injected into each oocyte. Injected cells were kept in ND96 solution (in mM): 96 $\mathrm{NaCl}, 2 \mathrm{KCl}, 1.8 \mathrm{CaCl}_{2}, 1 \mathrm{MgCl}_{2}, 5$ HEPES, $2.5 \mathrm{CH}_{3} \mathrm{COCO}_{2} \mathrm{Na}, 1: 100$ Pen-Strep, pH 7.6) at $18^{\circ} \mathrm{C}$ for 2-6 days for electrophysiology recordings.

$\underline{\text { Two-electrode voltage clamp (TEVC) and voltage-clamp fluorometry (VCF) }}$

Microelectrodes (Sutter Instrument, Item \#: B150-117-10) were made with a Sutter (P97) puller with resistances between $0.5 \mathrm{M} \Omega$ and $3 \mathrm{M} \Omega$ when filled with $3 \mathrm{M} \mathrm{KCl}$. After channel expression, oocyte cells were transferred to the recording chamber in ND96 bath solutions for whole-oocyte currents recording. Currents, sampled at $1 \mathrm{kHz}$ and low-pass-filtered at $2 \mathrm{kHz}$, were recorded using a CA-1B amplifier (Dagan, Minneapolis, $\mathrm{MN}$ ) with Patchmaster (HEKA) acquisition software. All recordings were performed at room temperature $\left(21-23^{\circ} \mathrm{C}\right.$ ). For ML277 experiments, ML277 stock (Sigma Aldrich, 1 $\mathrm{mM}$ in DMSO) was added to the bath and diluted to $1 \mu \mathrm{M}$. For the ion permeation experiments, $100 \mathrm{mM} \mathrm{K}^{+}$and $100 \mathrm{mM} \mathrm{Rb}^{+}$solutions $(96 \mathrm{mM} \mathrm{NaCl}$ was replaced with $100 \mathrm{mM} \mathrm{KCl}$ and $100 \mathrm{mM} \mathrm{RbCl}$ from ND96 solution) were perfused onto cells to steady state. 
For VCF experiments, oocytes were incubated in $10 \mu \mathrm{M}$ Alexa 488 C5-maleimide (Molecular Probes, Eugene, OR) on ice for $30 \mathrm{~min}$. To facilitate the labeling, Alexa 488 was prepared into high $\mathrm{K}^{+}$solution in $\mathrm{mM}\left(98 \mathrm{KCl}, 1.8 \mathrm{CaCl}_{2}, 5 \mathrm{HEPES}, \mathrm{pH} 7.6\right)$ to depolarize the membrane voltage so that the VSD could undergo outward movement. After 30 min, oocytes were transferred to normal ND96 solution and washed three times before recording. The excitation and emission lights were filtered for Alexa 488. During recording, the fluorescence signals from the VSD movements were collected by a photodiode (Pin20A, OSI Optoelectronics). The signals, sampled at $1 \mathrm{kHz}$ and filtered at $200 \mathrm{~Hz}$, were then amplified by an EPC10 (HEKA) amplifier and synchronized by the CA-1B amplifier.

\section{Electrophysiology Data analysis}

Data were analyzed with Clampfit (Axon Instruments, Inc., Sunnyvale, CA), Sigmaplot (SPSS, Inc., San Jose, CA), and IGOR (Wavemetrics, Lake Oswego, OR). Because of photo-bleaching, fluorescence signals were baseline subtracted by fitting the first $2 \mathrm{~s}$ signals at the $-80 \mathrm{mV}$ holding potential. Fluorescence-voltage $(\mathrm{F}-\mathrm{V})$ relationships were derived by normalizing the $\Delta F / F$ value at the end of each test pulse to the maximal value. $\mathrm{G}-\mathrm{V}$ and $\mathrm{F}-\mathrm{V}$ curves were fitted with either one or double Boltzmann equations in the form of $1 /\left(1+\exp \left(-z^{*} F^{*}\left(V-V_{1 / 2}\right) / R T\right)\right)$, where $V$ is the voltage, $z$ is the equivalent valence, $V_{1 / 2}$ is the half-maximal voltage, $F$ is the Faraday constant, $R$ is the gas constant, and $T$ is the absolute temperature. KCNQ1 tail currents were fitted with a double exponential function $F(t)=A_{1} \times \exp \left(-t / \tau_{1}\right)+A_{2} \times \exp \left(-t / \tau_{2}\right)+C$ as previously described (Hou et al., 2017) to quantify the hook currents $\left(A_{2}\right)$. For activation time constants $\left(\tau_{f}\right.$ and $\tau_{s}$ ) of KCNQ1 currents in Figure 2, KCNQ1 activation currents were fitted with a double exponential function to get the $\tau_{f}$ and $\tau_{s}$ for control, and were fitted separately with a single exponential function to get the $\tau_{\mathrm{f}}$ and $\tau_{\mathrm{s}}$ for currents after adding ML277.

\section{Kinetic modeling of ML277 effects}

The five-state kinetic model of $\mathrm{K}_{\mathrm{V}} 7.1$ channel was constructed from our previous sixstate model (Hou et al., 2017). The upper row RC, IC, AC stand for the VSD states at 
resting, intermediate, and activated when the pore is closed, and the lower row $\mathrm{IO}$ and $\mathrm{AO}$ stand for pore opening at intermediate and activated states. The voltage independent opening state "resting open" (RO) was deleted from the six-state model based on the rare transition from RC to RO (Hou et al., 2017). This simplified five-state model is capable to recapitulate the main characteristics of the $\mathrm{K}_{\mathrm{v}} 7.1$ two open states gating (Hou et al., 2017). We use the same Markov process to model the transition between each state, where $\alpha_{1}=a_{1}{ }^{*} \exp (v / m), \beta_{1}=c_{1}{ }^{*} \exp (-v / n), \alpha_{i}=a_{i}{ }^{*} \exp (v / b)$ and $\beta_{i}=$ $\mathrm{C}_{\mathrm{i}}{ }^{*} \exp (-\mathrm{v} / \mathrm{d})(\mathrm{i}=2$ and 3$)$ are the voltage-dependent rates of transitions. $\mathrm{k}_{1-4}$ are the E-M coupling rates (constant), where $\mathrm{k}_{2}$, i.e., the $\mathrm{E}-\mathrm{M}$ coupling rate from the $\mathrm{AO}$ state to the AC state, can be set to different values to simulate the control and after ML277 results. Reducing the value of $k_{2}$ by half can mimic the ML277 effects on KCNQ1 channel. The values of the parameters are as follows: $\mathrm{a}_{1}=0.00070 \mathrm{~ms}^{-1}, \mathrm{a}_{2}=0.0047 \mathrm{~ms}^{-1}, \mathrm{a}_{3}=0.15$ $\mathrm{ms}^{-1}, \mathrm{c}_{1}=0.0020 \mathrm{~ms}^{-1}, \mathrm{c}_{2}=0.00017 \mathrm{~ms}^{-1}, \mathrm{c}_{3}=0.048 \mathrm{~ms}^{-1}, \mathrm{~m}=46.0 \mathrm{mV}, \mathrm{n}=31.2 \mathrm{mV}, \mathrm{b}=$ $37.7 \mathrm{mV}, \mathrm{d}=41.5 \mathrm{mV}, \mathrm{k}_{1}=0.89, \mathrm{k}_{2}=853.08$ (for control), and $\mathrm{k}_{2}=426.54$ (for adding ML277), $\mathrm{k}_{3}=0.96, \mathrm{k}_{4}=103.82$. To balance the model, $\mathrm{c}_{3}=0.024 \mathrm{~ms}^{-1}$ for adding ML277. Kinetic parameters were optimized with CeL software (HUST, Wuhan, Hubei, China) as previously described (Hou et al., 2014; Wang et al., 2013). The reversal potential was set to $-80 \mathrm{mV}$, and the single-channel conductance was set to $0.18 \mathrm{pS}$ for KCNQ1 channels (Hou et al., 2017). 


\section{Acknowledgments}

We thank Po Wei Kang for reading and revising the manuscript. We also thank Prof. Jeanne Nerbonne for helpful discussion. This work was supported by R01 NS092570 and R01 HL126774 (to J.C.), and by AHA 18POST34030203 (to P.H.).

\section{Disclosures}

J.S. and J.C. are cofounders of a startup company VivoCor LLC, which is targeting $I_{\mathrm{Ks}}$ for the treatment of cardiac arrhythmia.

\section{Author Contributions}

P.H. conceived this work, and J.C. directed this work. P.H. conducted the voltage clamp and VCF experiments, J.S. and K.M.W. performed the molecular biology, P.H. and Y.G. performed the kinetic modeling of ML277. P.H. and J.C. wrote the paper with input from all authors. 


\section{Figures}

A

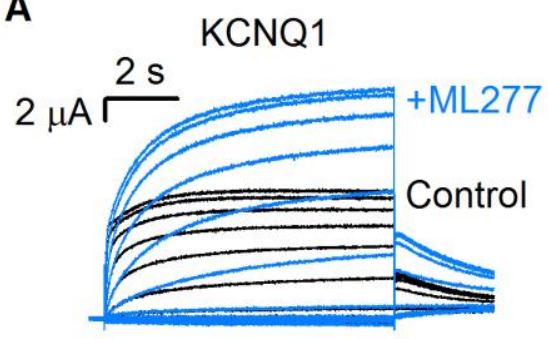

B

KCNQ1+KCNE1

$$
\text { Q1:E1=300:1 Q1:E1=4:1 }
$$

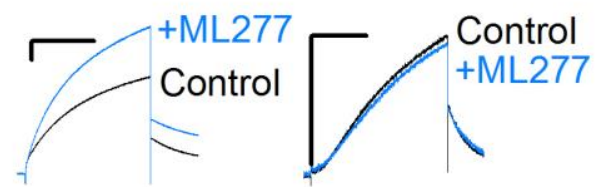

C
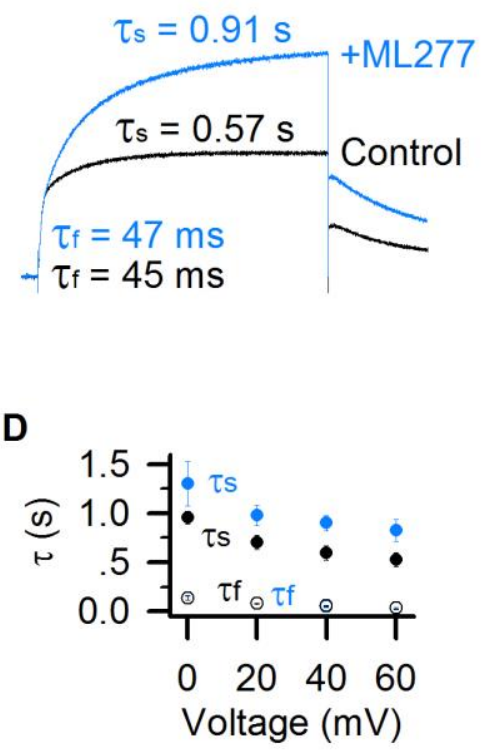

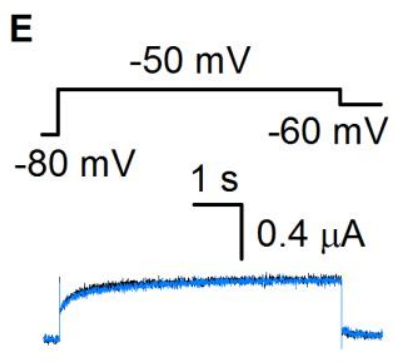

$\mathbf{F}$

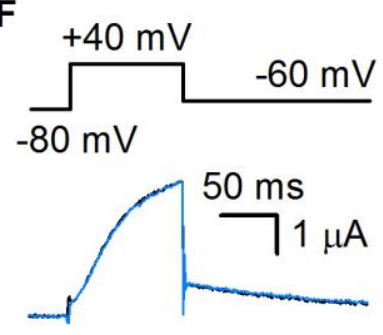

Figure 1. ML277 specifically changes the slow component of KCNQ1 currents. (A) KCNQ1 currents from Xenopus oocytes recorded before (black) and after (blue) adding $1 \mu \mathrm{M}$ ML277. The test voltages were from $-120 \mathrm{mV}$ to $80 \mathrm{mV}$ with $20 \mathrm{mV}$ increments, and then returned to $-40 \mathrm{mV}$ to measure the tail currents. (B) $1 \mu \mathrm{M}$ ML277 effects on KCNQ1+KCNE1 channels with injected mRNA weight ratio of KCNQ1:KCNE1=300:1 (left) and 4:1 (right). The test voltages were $40 \mathrm{mV}$ for $4 \mathrm{~s}$, and then returned to $-40 \mathrm{mV}$ to measure the tail currents. (C) Activation time constants $(\tau)$ of KCNQ1 currents recorded at $+40 \mathrm{mV}$ before (black) and after (blue) adding ML277. The time constants of the fast component $\left(\tau_{\mathrm{f}}\right)$ and slow component $\left(\tau_{\mathrm{s}}\right)$ were $45 \mathrm{~ms}$ and $0.57 \mathrm{~s}$ for control, and $47 \mathrm{~ms}$ and $0.91 \mathrm{~s}$ for after adding ML277. (D) Averaged results of activation time constants $\left(\tau_{f}\right.$ and $\left.\tau_{s}\right)$ of KCNQ1 currents recorded at different voltages before (black) and after (blue) adding ML277. $n \geq 4$. (E-F) KCNQ1 currents recorded before (black) and after (blue) adding ML277 with $5 \mathrm{~s}$ time duration at $-50 \mathrm{mV}$ (C) and $100 \mathrm{~ms}$ time duration at $+40 \mathrm{mV}(\mathrm{D})$. The tail currents were recorded at $-60 \mathrm{mV}$. Voltage protocols are shown on the top. 
A

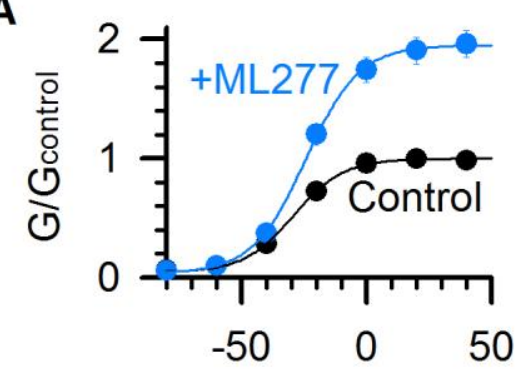

Voltage $(\mathrm{mV})$

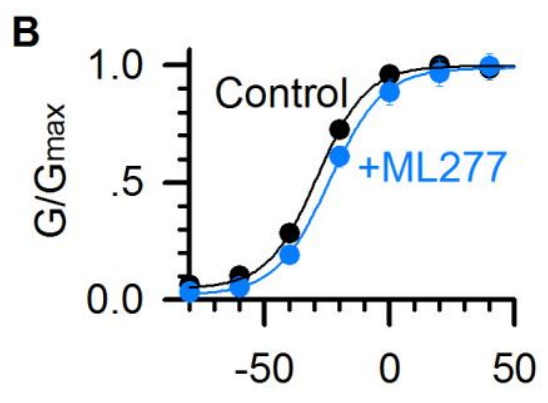

Voltage $(\mathrm{mV})$

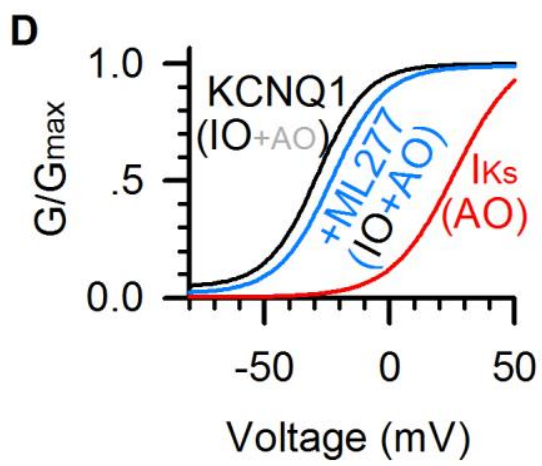

Figure 2. ML277 right shifts the G-V relationship of KCNQ1 channels. (A) G-V relationships of KCNQ1 channels before (black) and after (blue) adding 1 $\mu \mathrm{M} \mathrm{ML277.}$ Data points were fitted with a Boltzmann function. (B) Normalized G-V relationships from panel A. (C) Averaged results of $V_{50}$ of $G-V$ relations (voltage at which the $G-V$ is half maximal). The $\mathrm{V}_{50}$ for $\mathrm{KCNQ} 1$ channels is $-26.8 \pm 0.6 \mathrm{mV}$ in control (black), and $20.3 \pm 0.6 \mathrm{mV}$ after adding ML277 (blue), With a significant difference $\left(\mathrm{p}=5 \times 10^{-10}\right.$, paired $t$ test. $n=25)$. The $V_{50}$ of $I_{K s}$ channels (KCNQ1+KCNE1) $(25.6 \pm 3.1 \mathrm{mV}$, red) is also for comparison. (D) G-V relationships of the KCNQ1 channels before (black) and after (blue) adding $1 \mu \mathrm{M}$ ML277, and of $\mathrm{I}_{\mathrm{Ks}}$ channels (red). IO/AO composition in each type of channels is indicated in parentheses. All error bars in this and other figures are \pm SEM. 


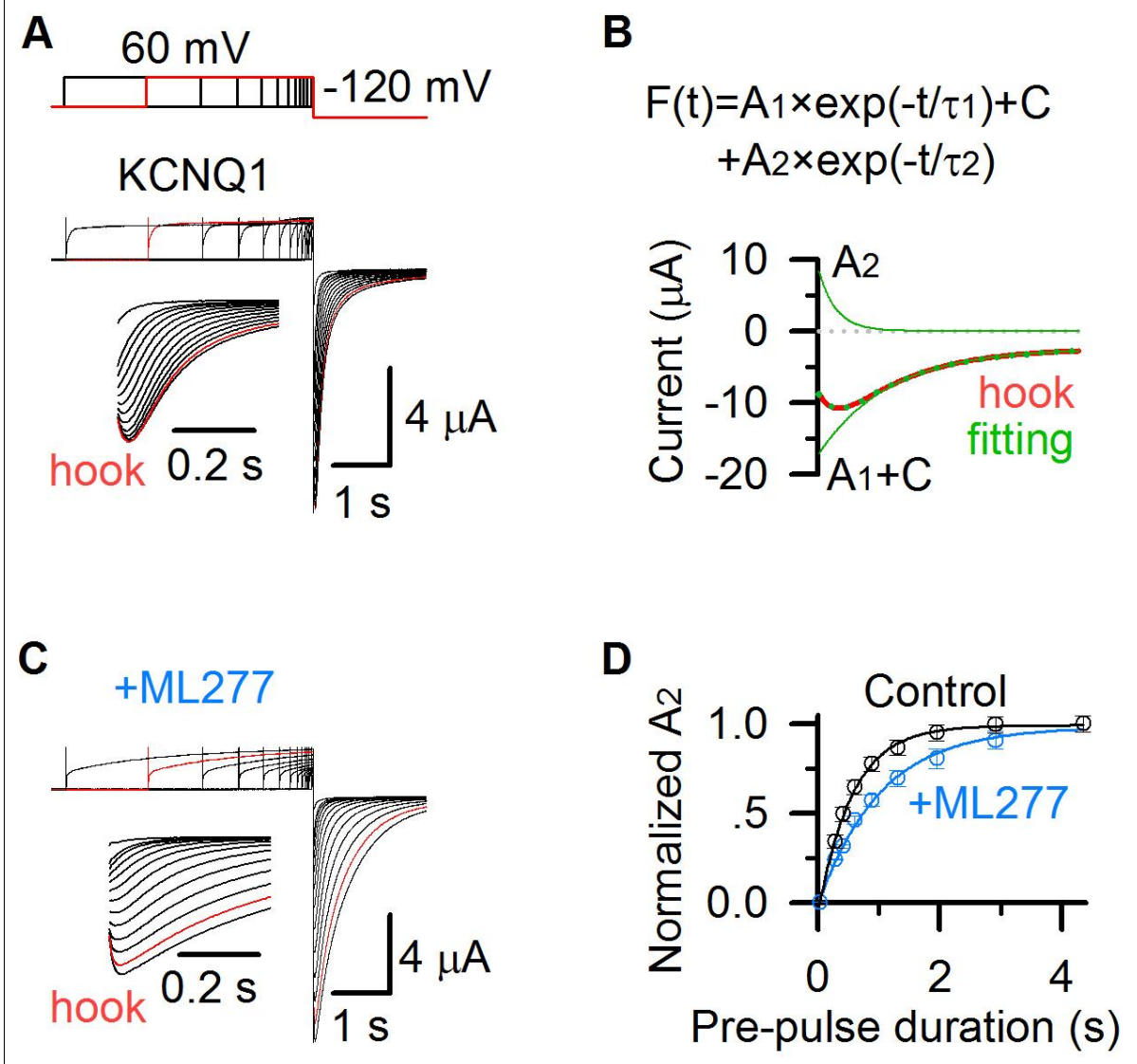

Figure 3. ML277 changes inactivation of KCNQ1 channels. (A) KCNQ1 tail currents recorded with pre-pulses of increasing time duration in $100 \mathrm{mM} \mathrm{K}^{+}$solution. The prepulses were $+60 \mathrm{mV}$ with time durations ranging between 0.03 and $4.355 \mathrm{~s}$, and the test pulse was $-120 \mathrm{mV}$ for $2 \mathrm{~s}$. The inset shows the hook in tail currents with an expanded time scale. The current recorded with a $2.913 \mathrm{~s}$ pre-pulse duration is shown in red. (B) KCNQ1 tail current recorded with a $2.913 \mathrm{~s}$ pre-pulse duration (red) was fitted with a double exponential function $F(t)=A_{1} \times \exp \left(-t / \tau_{1}\right)+A_{2} \times \exp \left(-t / \tau_{2}\right)+C$ (Hou et al., 2017). The fitting curve is shown in dotted green line, the deactivation component $\left(A_{1} \times \exp \left(-t / \tau_{1}\right)+C\right)$ and hook component $\left(A_{2} \times \exp \left(-t / \tau_{2}\right)\right)$ are shown in green lines. (C) KCNQ1 tail currents after applying ML277 recorded with pre-pulses of increasing time durations in $100 \mathrm{mM} \mathrm{K}^{+}$solution, with the same voltage protocol shown as in panel A. The inset shows the hook in tail currents with an expanded time scale. The current recorded with a $2.913 \mathrm{~s}$ pre-pulse duration is shown in red. (D) Normalized $A_{2}$ of KCNQ1 hook currents before (black) and after (blue) adding ML277 vs. time durations of the pre-pulse $(n \geq 4)$. Data are fitted with a single exponential function. 
A

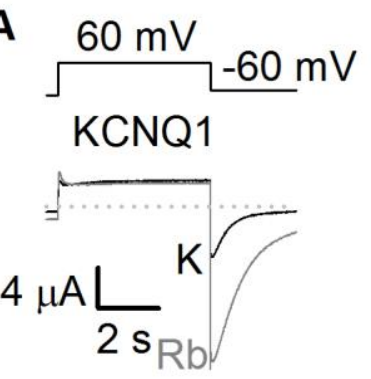

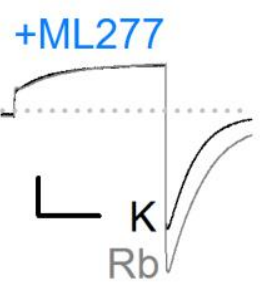

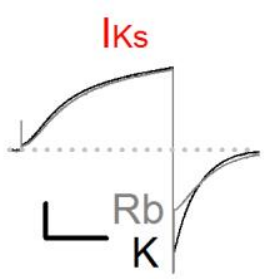

B

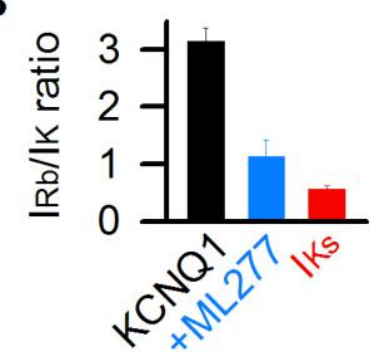

Figure 4. ML277 changes ion permeation of KCNQ1 channels. (A) Currents from KCNQ1 channels before (left) and after (middle) adding $1 \mu \mathrm{M}$ ML277, as well as $\mathrm{I}_{\mathrm{Ks}}$ channels (KCNQ1+KCNE1) (right) recorded in $100 \mathrm{mM} \mathrm{K}^{+}$(black) or $100 \mathrm{mM} \mathrm{Rb}^{+}$(gray) external solutions. The tail currents were elicited at $-60 \mathrm{mV}$ for $3 \mathrm{~s}$ from a pre-pulse of $+60 \mathrm{mV}$ for $5 \mathrm{~s}$ (top of left). (B) Averaged $\mathrm{Rb}^{+} / \mathrm{K}^{+}$permeability ratios of tail current amplitudes for KCNQ1 before (3.1 \pm 0.2 , black) and after (1.1 \pm 0.3$)$ adding ML277, and for $I_{\mathrm{Ks}}$ channels $(0.6 \pm 0.1$, red). All $n \geq 4$. 
A
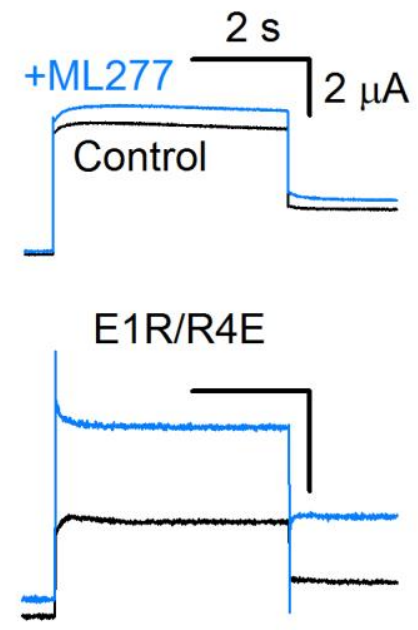

B

S338F

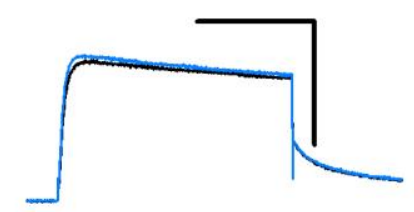

F351A

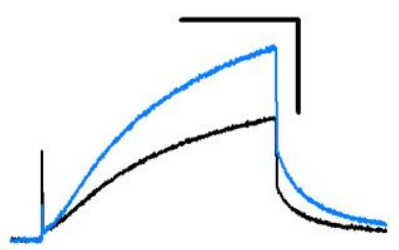

C

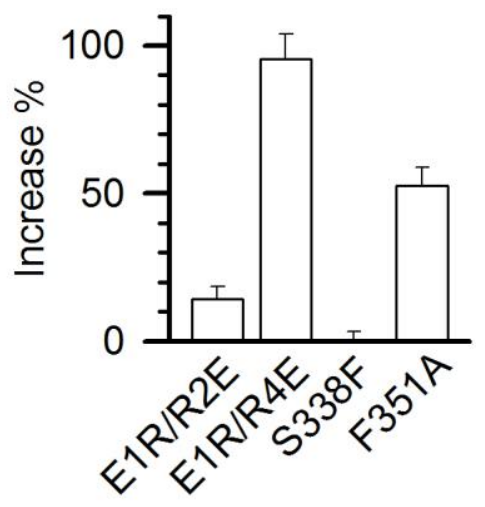

Figure 5. ML277 specifically increases the currents of mutant KCNQ1 channels that open only to the AO state. (A-B) $1 \mu \mathrm{M}$ ML277 effects on E1R/R2E, E1R/R4E, $\mathrm{S} 338 \mathrm{~F}$, and F351A mutant KCNQ1 channels. Currents were elicited at $+40 \mathrm{mV}$ for $4 \mathrm{~s}$ and then returned to $-40 \mathrm{mV}$ to record the tail currents. (C) Averaged percentage of

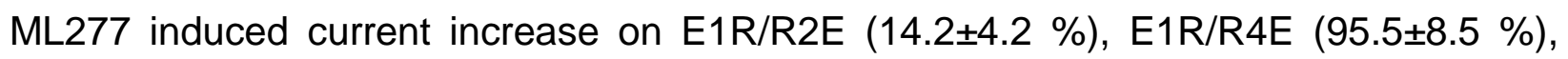

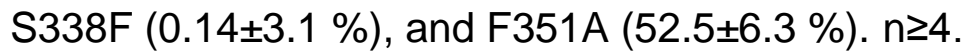




\section{A}
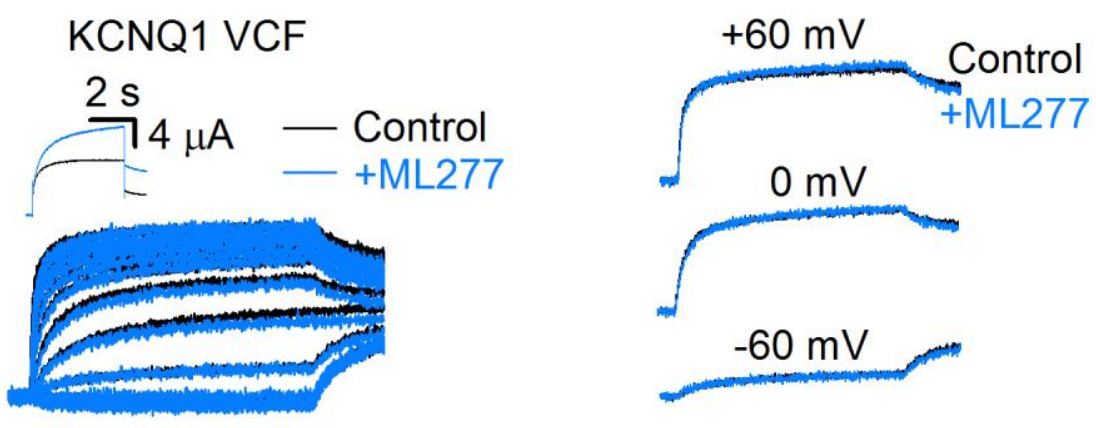

B

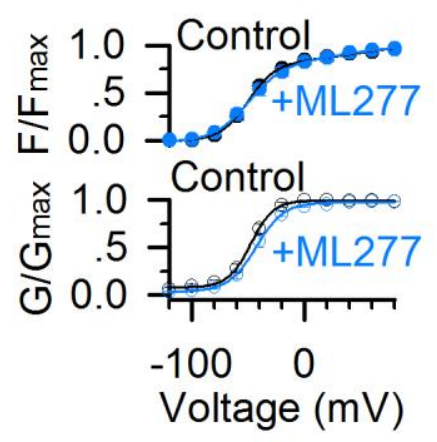

C

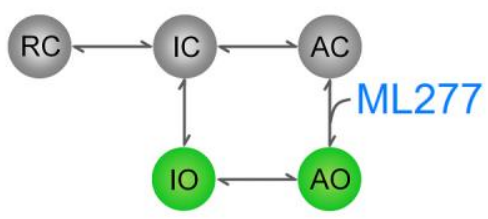

Figure 6. ML277 enhances currents of KCNQ1 channels but does not change VSD activation. (A) Fluorescence signals showing voltage sensor activation of pseudo-WT KCNQ1 channel (KCNQ1-C214A/G219C/C331A) before (black) and after (blue) adding $1 \mu \mathrm{M}$ ML277. The signals after applying ML277 were normalized to the peak from the control to correct the effects of photobleaching. Inset shows current traces of pseudoWT KCNQ1 channel before (black) and after (blue) adding ML277 at $+40 \mathrm{mV}$. Right panels show VCF results of pseudo-WT KCNQ1 channel before (black) and after (blue) adding ML277 recorded at $-60 \mathrm{mV}, 0 \mathrm{mV}$, and $60 \mathrm{mV}$. (B) Fluorescence-voltage ( $\mathrm{F}-\mathrm{V}$, $n=4)$ and $G-V(n=3)$ relationships of pseudo-WT KCNQ1 before (black) and after (blue) adding ML277. (C) A cartoon scheme to illustrate that ML277 specifically enhances E-M coupling when the VSD is at the activated state. 
A

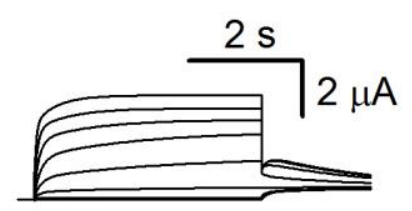

B

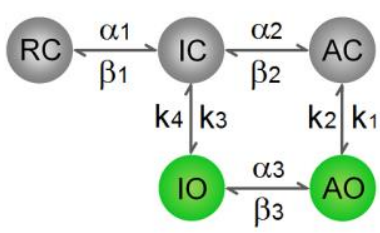

\begin{tabular}{c|l}
\hline Control & $+\mathrm{ML} 277$ \\
\hline $\mathrm{k} 2$ & $\mathrm{k} 2 / 2, \beta 3 / 2$ \\
\hline
\end{tabular}
$+\mathrm{ML} 277$

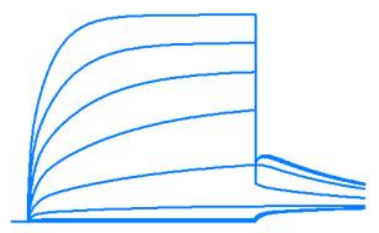

C

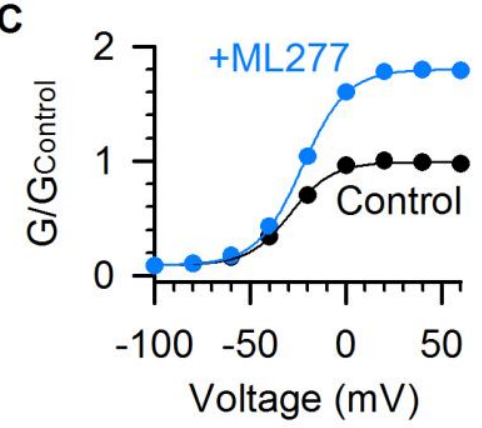

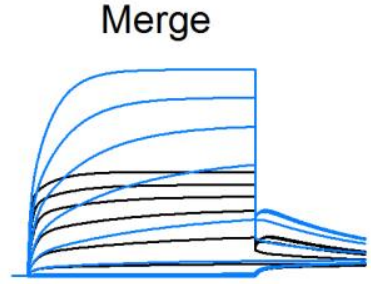

D

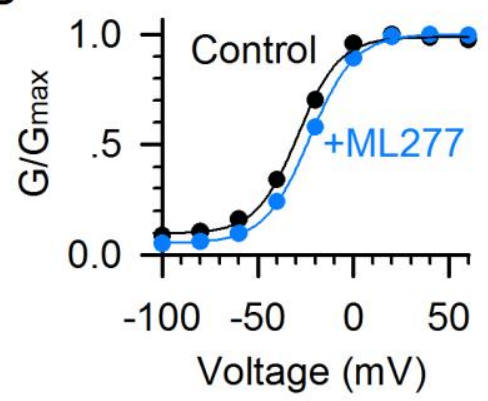

Figure 7. Model simulations of ML277 effects on KCNQ1 channels. (A) Model simulations of KCNQ1 activation currents before (black) and after (blue) adding ML277. (B) Five-state markov model to show the two open states gating processes of KCNQ1 channel. $\alpha$ and $\beta$ are voltage-dependent transitions. $k_{1-4}$ are E-M coupling rates (constant). Reducing the value of $k_{2}$ by half $\left(k_{2}=853.08\right.$ for control, and $k_{2}=426.54$ for adding ML277) can mimic the ML277 effects on KCNQ1 channel. To balance the model, $\beta_{3}$ is also reduced by half for adding ML277 (see Methods). (C-D) G-V relationships of simulated KCNQ1 before (black) and after (blue) adding ML277. Data points were fitted with a Boltzmann function. 


\section{References}

Abitbol, I., Peretz, A., Lerche, C., Busch, A.E., and Attali, B. (1999). Stilbenes and fenamates rescue the loss of I(KS) channel function induced by an LQT5 mutation and other IsK mutants. The EMBO journal 18, 4137-4148.

Baker, O.S., Larsson, H.P., Mannuzzu, L.M., and Isacoff, E.Y. (1998). Three transmembrane conformations and sequence-dependent displacement of the S4 domain in shaker $\mathrm{K}+$ channel gating. Neuron 20, 1283-1294.

Barhanin, J., Lesage, F., Guillemare, E., Fink, M., Lazdunski, M., and Romey, G. (1996). $\mathrm{K}(\mathrm{V}) \mathrm{LQT1}$ and IsK (minK) proteins associate to form the I(Ks) cardiac potassium current. Nature 384, 78-80.

Barro-Soria, R., Perez, M.E., and Larsson, H.P. (2015). KCNE3 acts by promoting voltage sensor activation in KCNQ1. Proceedings of the National Academy of Sciences of the United States of America 112, E7286-7292.

Barro-Soria, R., Rebolledo, S., Liin, S.I., Perez, M.E., Sampson, K.J., Kass, R.S., and Larsson, H.P. (2014). KCNE1 divides the voltage sensor movement in KCNQ1/KCNE1 channels into two steps. Nature communications 5, 3750.

Bezanilla, F., Perozo, E., and Stefani, E. (1994). Gating of Shaker K+ channels: II. The components of gating currents and a model of channel activation. Biophysical journal 66, 1011-1021.

Boulet, I.R., Labro, A.J., Raes, A.L., and Snyders, D.J. (2007). Role of the S6 Cterminus in KCNQ1 channel gating. The Journal of physiology 585, 325-337.

Busch, A.E., Busch, G.L., Ford, E., Suessbrich, H., Lang, H.J., Greger, R., Kunzelmann, K., Attali, B., and Stuhmer, W. (1997). The role of the IsK protein in the specific pharmacological properties of the IKs channel complex. British journal of pharmacology 122, 187-189.

Carvalho-de-Souza, J.L., and Bezanilla, F. (2018). Nonsensing residues in S3-S4 linker's $\mathrm{C}$ terminus affect the voltage sensor set point in $\mathrm{K}(+)$ channels. The Journal of general physiology 150, 307-321.

Chiamvimonvat, N., Chen-Izu, Y., Clancy, C.E., Deschenes, I., Dobrev, D., Heijman, J., Izu, L., Qu, Z., Ripplinger, C.M., Vandenberg, J.I., et al. (2017). Potassium currents in the heart: functional roles in repolarization, arrhythmia and therapeutics. The Journal of physiology 595, 2229-2252.

Cruickshank, S.F., Baxter, L.M., and Drummond, R.M. (2003). The Cl(-) channel blocker niflumic acid releases $\mathrm{Ca}(2+)$ from an intracellular store in rat pulmonary artery smooth muscle cells. British journal of pharmacology 140, 1442-1450.

Cui, J. (2016). Voltage-Dependent Gating: Novel Insights from KCNQ1 Channels. Biophysical journal 110, 14-25. 
De Silva, A.M., Manville, R.W., and Abbott, G.W. (2018). Deconstruction of an African folk medicine uncovers a novel molecular strategy for therapeutic potassium channel activation. Science advances 4, eaav0824.

Delemotte, L., Tarek, M., Klein, M.L., Amaral, C., and Treptow, W. (2011). Intermediate states of the Kv1.2 voltage sensor from atomistic molecular dynamics simulations. Proceedings of the National Academy of Sciences of the United States of America 108, 6109-6114.

Gao, H., Boillat, A., Huang, D., Liang, C., Peers, C., and Gamper, N. (2017). Intracellular zinc activates $\mathrm{KCNQ}$ channels by reducing their dependence on phosphatidylinositol 4,5-bisphosphate. Proceedings of the National Academy of Sciences of the United States of America 114, E6410-E6419.

Gao, Z., Xiong, Q., Sun, H., and Li, M. (2008). Desensitization of chemical activation by auxiliary subunits: convergence of molecular determinants critical for augmenting KCNQ1 potassium channels. The Journal of biological chemistry 283, 22649-22658.

Gibor, G., Yakubovich, D., Rosenhouse-Dantsker, A., Peretz, A., Schottelndreier, H., Seebohm, G., Dascal, N., Logothetis, D.E., Paas, Y., and Attali, B. (2007). An inactivation gate in the selectivity filter of KCNQ1 potassium channels. Biophysical journal 93, 4159-4172.

Glauner, K.S., Mannuzzu, L.M., Gandhi, C.S., and Isacoff, E.Y. (1999). Spectroscopic mapping of voltage sensor movement in the Shaker potassium channel. Nature 402, 813-817.

Guo, S., Chen, Y., Pang, C., Wang, X., Qi, J., Mo, L., Zhang, H., An, H., and Zhan, Y. (2017). Ginsenoside Rb1, a novel activator of the TMEM16A chloride channel, augments the contraction of guinea pig ileum. Pflugers Archiv : European journal of physiology 469, 681-692.

Hill, A.P., and Sitsapesan, R. (2002). DIDS modifies the conductance, gating, and inactivation mechanisms of the cardiac ryanodine receptor. Biophysical journal 82 , 3037-3047.

Hoosien, M., Ahearn, M.E., Myerburg, R.J., Pham, T.V., Miller, T.E., Smets, M.J., Baumbach-Reardon, L., Young, M.L., Farooq, A., and Bishopric, N.H. (2013). Dysfunctional potassium channel subunit interaction as a novel mechanism of long QT syndrome. Heart rhythm 10, 728-737.

Hou, P., Eldstrom, J., Shi, J., Zhong, L., McFarland, K., Gao, Y., Fedida, D., and Cui, J. (2017). Inactivation of KCNQ1 potassium channels reveals dynamic coupling between voltage sensing and pore opening. Nature communications 8, 1730.

Hou, P., Zhang, R., Liu, Y., Feng, J., Wang, W., Wu, Y., and Ding, J. (2014). Physiological role of Kv1.3 channel in T lymphocyte cell investigated quantitatively by kinetic modeling. PloS one 9, e89975. 
Jensen, M.O., Jogini, V., Borhani, D.W., Leffler, A.E., Dror, R.O., and Shaw, D.E. (2012). Mechanism of voltage gating in potassium channels. Science 336, 229-233.

Keating, M.T., and Sanguinetti, M.C. (2001). Molecular and cellular mechanisms of cardiac arrhythmias. Cell 104, 569-580.

Lacroix, J.J., Pless, S.A., Maragliano, L., Campos, F.V., Galpin, J.D., Ahern, C.A., Roux, B., and Bezanilla, F. (2012). Intermediate state trapping of a voltage sensor. The Journal of general physiology 140, 635-652.

Larsen, A.P., Steffensen, A.B., Grunnet, M., and Olesen, S.P. (2011). Extracellular potassium inhibits Kv7.1 potassium channels by stabilizing an inactivated state. Biophysical journal 101, 818-827.

Larsson, H.P., Baker, O.S., Dhillon, D.S., and Isacoff, E.Y. (1996). Transmembrane movement of the shaker K+ channel S4. Neuron 16, 387-397.

Ledwell, J.L., and Aldrich, R.W. (1999). Mutations in the S4 region isolate the final voltage-dependent cooperative step in potassium channel activation. The Journal of general physiology 113, 389-414.

Liu, R., Zhang, Z., Liu, H., Hou, P., Lang, J., Wang, S., Yan, H., Li, P., Huang, Z., Wu, H., et al. (2013). Human beta-defensin 2 is a novel opener of Ca2+-activated potassium channels and induces vasodilation and hypotension in monkeys. Hypertension 62, 415425.

Ma, D., Wei, H., Lu, J., Huang, D., Liu, Z., Loh, L.J., Islam, O., Liew, R., Shim, W., and Cook, S.A. (2015). Characterization of a novel KCNQ1 mutation for type 1 long QT syndrome and assessment of the therapeutic potential of a novel IKs activator using patient-specific induced pluripotent stem cell-derived cardiomyocytes. Stem cell research \& therapy 6, 39.

Mattmann, M.E., Yu, H., Lin, Z., Xu, K., Huang, X., Long, S., Wu, M., McManus, O.B., Engers, D.W., Le, U.M., et al. (2012). Identification of (R)-N-(4-(4methoxyphenyl)thiazol-2-yl)-1-tosylpiperidine-2-carboxamide, ML277, as a novel, potent and selective $\mathrm{K}(\mathrm{v}) 7.1$ (KCNQ1) potassium channel activator. Bioorganic \& medicinal chemistry letters 22, 5936-5941.

Mruk, K., and Kobertz, W.R. (2009). Discovery of a novel activator of KCNQ1-KCNE1 K channel complexes. PloS one 4, e4236.

Nakagawa, H., Munakata, T., and Sunami, A. (2019). Mexiletine Block of Voltage-Gated Sodium Channels: Isoform- and State-Dependent Drug-Pore Interactions. Molecular pharmacology 95, 236-244.

Nakajo, K., Ulbrich, M.H., Kubo, Y., and Isacoff, E.Y. (2010). Stoichiometry of the KCNQ1 - KCNE1 ion channel complex. Proceedings of the National Academy of Sciences of the United States of America 107, $18862-18867$. 
Osteen, J.D., Barro-Soria, R., Robey, S., Sampson, K.J., Kass, R.S., and Larsson, H.P. (2012). Allosteric gating mechanism underlies the flexible gating of KCNQ1 potassium channels. Proceedings of the National Academy of Sciences of the United States of America 109, 7103-7108.

Osteen, J.D., Gonzalez, C., Sampson, K.J., lyer, V., Rebolledo, S., Larsson, H.P., and Kass, R.S. (2010). KCNE1 alters the voltage sensor movements necessary to open the KCNQ1 channel gate. Proc Natl Acad Sci U S A 107, 22710-22715.

Pusch, M., Bertorello, L., and Conti, F. (2000). Gating and flickery block differentially affected by rubidium in homomeric KCNQ1 and heteromeric KCNQ1/KCNE1 potassium channels. Biophysical journal 78, 211-226.

Pusch, M., Magrassi, R., Wollnik, B., and Conti, F. (1998). Activation and inactivation of homomeric KvLQT1 potassium channels. Biophysical journal 75, 785-792.

Restier, L., Cheng, L., and Sanguinetti, M.C. (2008). Mechanisms by which atrial fibrillation-associated mutations in the S1 domain of KCNQ1 slow deactivation of IKs channels. The Journal of physiology 586, 4179-4191.

Salata, J.J., Jurkiewicz, N.K., Wang, J., Evans, B.E., Orme, H.T., and Sanguinetti, M.C. (1998). A novel benzodiazepine that activates cardiac slow delayed rectifier K+ currents. Molecular pharmacology 54, 220-230.

Sanguinetti, M.C., Curran, M.E., Zou, A., Shen, J., Spector, P.S., Atkinson, D.L., and Keating, M.T. (1996). Coassembly of K(V)LQT1 and minK (IsK) proteins to form cardiac I(Ks) potassium channel. Nature 384, 80-83.

Schwartz, P.J., Crotti, L., and Insolia, R. (2012). Long-QT syndrome: from genetics to management. Circulation Arrhythmia and electrophysiology 5, 868-877.

Seebohm, G., Pusch, M., Chen, J., and Sanguinetti, M.C. (2003a). Pharmacological activation of normal and arrhythmia-associated mutant KCNQ1 potassium channels. Circulation research 93, 941-947.

Seebohm, G., Sanguinetti, M.C., and Pusch, M. (2003b). Tight coupling of rubidium conductance and inactivation in human KCNQ1 potassium channels. The Journal of physiology 552, 369-378.

Sigg, D., Stefani, E., and Bezanilla, F. (1994). Gating current noise produced by elementary transitions in Shaker potassium channels. Science 264, 578-582.

Sigworth, F.J. (1994). Voltage gating of ion channels. Quarterly reviews of biophysics 27, 1-40.

Silva, J.R., Pan, H., Wu, D., Nekouzadeh, A., Decker, K.F., Cui, J., Baker, N.A., Sept, D., and Rudy, Y. (2009). A multiscale model linking ion-channel molecular dynamics and electrostatics to the cardiac action potential. Proc Natl Acad Sci U S A 106, 1110211106. 
Silverman, W.R., Roux, B., and Papazian, D.M. (2003). Structural basis of two-stage voltage-dependent activation in $\mathrm{K}+$ channels. Proceedings of the National Academy of Sciences of the United States of America 100, 2935-2940.

Sun, J., and MacKinnon, R. (2017). Cryo-EM Structure of a KCNQ1/CaM Complex Reveals Insights into Congenital Long QT Syndrome. Cell 169, 1042-1050 e1049.

Tristani-Firouzi, M., and Sanguinetti, M.C. (1998). Voltage-dependent inactivation of the human $\mathrm{K}+$ channel KvLQT1 is eliminated by association with minimal $\mathrm{K}+$ channel (minK) subunits. The Journal of physiology 510 ( Pt 1), 37-45.

Wang, Q., Curran, M.E., Splawski, I., Burn, T.C., Millholland, J.M., VanRaay, T.J., Shen, J., Timothy, K.W., Vincent, G.M., de Jager, T., et al. (1996). Positional cloning of a novel potassium channel gene: KVLQT1 mutations cause cardiac arrhythmias. Nature genetics 12, 17-23.

Wang, W., Luo, J., Hou, P., Yang, Y., Xiao, F., Yuchi, M., Qu, A., Wang, L., and Ding, J. (2013). Native gating behavior of ion channels in neurons with null-deviation modeling. PloS one 8, e77105.

Wu, D., Delaloye, K., Zaydman, M.A., Nekouzadeh, A., Rudy, Y., and Cui, J. (2010a). State-dependent electrostatic interactions of $S 4$ arginines with $\mathrm{E} 1$ in $\mathrm{S} 2$ during Kv7.1 activation. The Journal of general physiology 135, 595-606.

Wu, D., Pan, H., Delaloye, K., and Cui, J. (2010b). KCNE1 remodels the voltage sensor of Kv7.1 to modulate channel function. Biophysical journal 99, 3599-3608.

Xiong, Q., Sun, H., and Li, M. (2007). Zinc pyrithione-mediated activation of voltagegated KCNQ potassium channels rescues epileptogenic mutants. Nature chemical biology 3, 287-296.

Xu, H., Li, T., Rohou, A., Arthur, C.P., Tzakoniati, F., Wong, E., Estevez, A., Kugel, C., Franke, Y., Chen, J., et al. (2019). Structural Basis of Nav1.7 Inhibition by a GatingModifier Spider Toxin. Cell 176, 1238-1239.

Xu, Y., Wang, Y., Zhang, M., Jiang, M., Rosenhouse-Dantsker, A., Wassenaar, T., and Tseng, G.N. (2015). Probing binding sites and mechanisms of action of an I(Ks) activator by computations and experiments. Biophysical journal 108, 62-75.

Yarotskyy, V., Gao, G., Du, L., Ganapathi, S.B., Peterson, B.Z., and Elmslie, K.S. (2010). Roscovitine binds to novel L-channel (CaV1.2) sites that separately affect activation and inactivation. The Journal of biological chemistry 285, 43-53.

Yazawa, M., Hsueh, B., Jia, X., Pasca, A.M., Bernstein, J.A., Hallmayer, J., and Dolmetsch, R.E. (2011). Using induced pluripotent stem cells to investigate cardiac phenotypes in Timothy syndrome. Nature 471, 230-234.

Yu, H., Lin, Z., Mattmann, M.E., Zou, B., Terrenoire, C., Zhang, H., Wu, M., McManus, O.B., Kass, R.S., Lindsley, C.W., et al. (2013). Dynamic subunit stoichiometry confers a progressive continuum of pharmacological sensitivity by KCNQ potassium channels. 
Proceedings of the National Academy of Sciences of the United States of America 110, 8732-8737.

Yu, H., Lin, Z., Xu, K., Huang, X., Long, S., Wu, M., McManus, O.B., Le Engers, J., Mattmann, M.E., Engers, D.W., et al. (2010). Identification of a novel, small molecule activator of KCNQ1 channels. In Probe Reports from the NIH Molecular Libraries Program (Bethesda (MD)).

Zagotta, W.N., Hoshi, T., and Aldrich, R.W. (1994). Shaker potassium channel gating. III: Evaluation of kinetic models for activation. The Journal of general physiology 103, 321362.

Zaydman, M.A., Kasimova, M.A., McFarland, K., Beller, Z., Hou, P., Kinser, H.E., Liang, H., Zhang, G., Shi, J., Tarek, M., et al. (2014). Domain-domain interactions determine the gating, permeation, pharmacology, and subunit modulation of the IKs ion channel. eLife 3, e03606.

Zaydman, M.A., Silva, J.R., Delaloye, K., Li, Y., Liang, H., Larsson, H.P., Shi, J., and Cui, J. (2013). Kv7.1 ion channels require a lipid to couple voltage sensing to pore opening. Proc Natl Acad Sci U S A 110, 13180-13185.

Zheng, Y., Zhu, X., Zhou, P., Lan, X., Xu, H., Li, M., and Gao, Z. (2012). Hexachlorophene is a potent KCNQ1/KCNE1 potassium channel activator which rescues LQTs mutants. PloS one 7, e51820.

Zhu, W., Mazzanti, A., Voelker, T.L., Hou, P., Moreno, J.D., Angsutararux, P., Naegle, K.M., Priori, S.G., and Silva, J.R. (2019). Predicting Patient Response to the Antiarrhythmic Mexiletine Based on Genetic Variation. Circulation research 124, 539552. 